\title{
The Use of Immunoglobulin in Bone Marrow Transplantation
}

\author{
PETER J. TUTSCHKA ${ }^{1}$
}

\begin{abstract}
The role of bone marrow transplantation is to restore lymphohematopoietic function of a recipient whose marrow has been destroyed, either by disease or by the preparative therapy employed in an attempt to eradicate the patient's lymphohematopoietic malignancy. The restoration of lymphohematopoietic function through the donor graft occurs in stages, requires several months, and is often not completed until 1 to 2 years after transplantation. These sequential steps of immunoreconstitution are associated with a number of definable and predictable immune deficiencies and seem to be responsible for the pattern of complications that emerges after transplantation. Most of these complications are either the result of, or associated with, infections that also occur in an almost predictable pattern. In the various phases of immune deficiency following sequentially after transplantation, the humoral immune system is greatly affected, thus raising the possibility that passively administered antibodies in the form of immune globulin therapy might be beneficial in all phases of the marrow transplant procedure. This paper attempts to summarize the use of immune globulin preparations in clinical bone marrow transplantation, showing the rationale for and some of the results of therapeutic immune globulin administration.
\end{abstract}

KEY WORDS: Cytomegalovirus (CMV); graft-vs-host disease (GVHD); interstitial pneumonia; intravenous immune globulin (IVIG); total-body irradiation (TBI).

Intravenous immune globulin (IVIG) is now widely used in bone marrow transplantation, despite the fact that critical randomized, controlled studies necessary to permit utilization on a scientific basis have largely not been done yet. This paper attempts to give an overview of the use of immune globulins and the rationale for their use in clinical marrow transplantation.

Prior to bone marrow transplantation the recipient's marrow must be completely eradicated. The hematopoietic system and the immune system are then rebuilt, almost in their entirety. This rebuilding of the lymphohematopoietic system does not occur immediately; instead, it goes through several separate, sequential phases which are associated with an almost predictable pattern of complications, especially infectious complications.

In the preengraftment phase, pancytopenia is the most important event. This is followed by an early postengraftment phase, characterized immunobiologically by acute graft-vs-host disease (GVHD) and severe combined immunodeficiency. The late postengraftment phase is characterized by chronic GVHD and immunodeficiency but of a more humoral type. Almost predictably we first see bacterial infections followed by nonbacterial, viral infections, particularly interstitial pneumonias. Later, there are some well-defined infections with encapsulated organisms, the latter sometimes even very late after bone marrow transplantation.

In the preengraftment phase there is complete aplasia with absolute neutropenia and a severe lymphopenia. Immunoglobulin levels decrease to less than $50 \%$ of normal about 3 weeks after bone marrow transplantation. The patient's defenses have also been weakened by severe impairment of the physical barrier functions due to the very aggressive preparative therapy necessary for bone marrow transplantation. This gives rise to infections derived primarily from the bacterial microflora reservoirs of the patient, such as gram-negative bacteria from the gastrointestinal tract and grampositive bacteria from the integument such as Staphylococcus epidermidis from the skin. In recent experience gram-positive bacteria have replaced gram-negative bacteria as the dominant sources of infection. In 15 to $20 \%$ of patients in the preengraftment phase bacteremia is present, usually associated with sepsis and circulatory collapse. 
There are several theoretical ways to prevent those early infections. First, one could try to develop less toxic preparative regimens so that the barrier function was less impaired. The preparative regimen that we developed in our center is one in which total-body irradiation (TBI) is replaced by a seemingly less toxic agent such as busulfan (1). Such a regimen permits a very short period of aplasia (for only 5 to 7 days), which markedly reduces the danger to the patient. Second, one could try to reduce the patient's own microflora reservoirs by providing a gnotobiotic, highly restricted environment. It has been shown that patients with aplastic anemia who were placed in such an environment had a significantly increased probability of survival following bone marrow transplantation (2). Third, one could try to bolster the humoral immunity by passively administering antibodies against the relevant microorganisms.

Commercially available immune globulin preparations do indeed contain antibodies against a wide range of pathogens and toxins, in fact against virtually all the pathogenic organisms that are important for infections in the transplant setting. The activation of the complement system by the administration of immune globulins will lead to increased phagocytosis and an increased granulocytochemotaxis. Immune globulins may also prevent bacterial attachment on the mucosal surfaces because the receptor sites will be occupied by the infused antibodies. Furthermore, some reasonably wellcontrolled studies have shown that passive immune globulin therapy is effective for burn patients as well as for trauma patients $(3,4)$.

In our own program we have tried to utilize all the accepted measures for infectious prophylaxis. We studied 110 consecutive transplant patients for whom we substituted busulfan in place of TBI. We used selective body decontamination in an ultraclean environment and administered IVIG at a dose of $500 \mathrm{mg} / \mathrm{kg}$ every 2 weeks for a total of 4 months. With this regimen the incidence of bacteremia was only $2.7 \%$ and almost half of the patients developed no fever during aplasia. Unfortunately, this study was not a controlled one and the important question if IVIG given systemically will reduce infectious complications during aplasia remains unanswered. Several placebo-controlled trials are under way in a number of transplant centers to resolve this matter.

From the viewpoint of the immunobiologist, phase II, the phase early after marrow engraftment, is the more important period. A number of critical immune events take place in this second phase. By this time host B-cell immunity has disappeared completely but donor B-cell immunity has not yet been reestablished. Adoptive T-cell donor immunity (due to mature cells that were transferred with the bone marrow) has disappeared as well. The function of the helper $T$ cells is markedly impaired, either because the stem cells have not yet developed into the appropriate mature $\mathrm{T}$ cells or because the patients are receiving immunosuppressive agents. The cytotoxic effector function is also impaired in these patients. In addition to these diffculties, the donor immune system must reorient itself in a different MHC-restriction setting. Furthermore, nonspecific suppressor cells will appear 30 to 50 days after transplantation, resulting in a global impairment of the immune defense system. Acute GVHD might be present, markedly worsening the already present immune defect in the second phase of transplantation. An oversimplified definition of GVHD describes it as an immune attack by donor $T$ lymphocytes on certain host target cells in the gastrointestinal tract, the liver, and particularly, the skin. The resultant cutaneous disease is characterized by discoloration and desquamation followed by severe erythema together with epidermal lysis in which the entire integument is affected. A number of infectious complications are associated with acute GVHD, especially those due to resistant gram-negative bacteria, but also Candida infections and gram-positive infections caused by Corynebacteria. Interstitial pneumonia, a very important infection which is often associated with acute GVHD, is discussed shortly.

There is an intricate relationship between GVHD and infections. Germ-free rodents which are transplanted in a germ-free environment will not develop GVHD even if they receive a mismatched transplant. Conversely, intentional contamination of these chimeric animals will cause GVHD (5). Patients in germ-free environments have a reduced incidence of severe GVHD. Furthermore, cutaneous GVHD will develop in areas which have tissue damage, especially if this damage is caused by a virus. Finally, viral infections are known to trigger GVHD in rats (6). Could IVIG have a role in preventing GVHD? Animal studies, including some from our laboratory, indicate that when antibody against Escherichia coli is given systemically to rats, it will reduce GVHD in matched bone marrow transplants. This suggests that IVIG might have some influence on GVHD. It is possible that IVIG 
could act as an immune modulator. An Fcdependent blockade of reticuloendothelial structures by IVIG has been described and studies in animal models have shown that immune globulins can induce antiidiotypic regulation. Also on the basis of animal models, we know that suppressorcell function can be restored with the use of IVIG. Since specific suppressor cells are important in counteracting GVHD, this is another instance in which IVIG may play a role, at least in theory. The most important effect of IVIG, however, might be an indirect one by which prevention of infections will, in turn, avert the trigger for GVHD. Although at the present time GVHD is no longer a major problem in most transplant programs, GVHDassociated viral infections are still of great importance.

The two major viral infections in phase II express themselves either as interstitial pneumonia or as a hemorrhagic gastroenteritis not unlike the necrotizing enterocolitis in the pediatric population which is described elsewhere in this issue. The interstitial infiltrate of the early pneumonia quickly spreads over both lungs, often resulting in a complete whitening of the lungs which is accompanied by severe aduilt respiratory disease syndrome (ARDS). In at least half the cases, cytomegalovirus (CMV) is associated with ARDS. The incidence of this disease varies between 15 and $40 \%$, with CMV being responsible for about half of the cases. When CMV is the agent, mortality can range from 85 to as much as $100 \%$.

There are several risk factors for the development of interstitial pneumonia such as TBI (if the lung dose exceeds 600 rads), acute GVHD, increased age of the patient, CMV seropositivity of the patient before the transplant, transfusion of blood products from CMV-positive donors, inability to generate an antiviral cytotoxicity (which is very common in the postengraftment phase), and inability to mount a humoral antibody response. The latter defect is quite important and suggests a potential role for passive therapy with antibodies. Historically bone marrow transplant recipients who have high titers of complement-fixing antibodies to CMV prior to transplant have had a better prognosis for overcoming such a severe viral pneumonia. In the murine model, it could be shown that passive immunization can modify CMV infection.

Several studies have been carried out to see if passive immunization with antibodies against $\mathrm{CMV}$ would prevent interstitial pneumonia. Investigators from UCLA, Minnesota, and Sloan-Kettering all showed, in reasonably well-controlled studies, that the incidence of CMV interstitial pneumonia was reduced in patients given high-titered antibody preparations compared to that in the control group. This finding triggered numerous other studies, mostly uncontrolled, which can be summarized as follows.

IVIG is effective in preventing $\mathrm{CMV}$ infections in $\mathrm{CMV}$-seronegative recipients who receive CMVseronegative grafts. It is likely to be ineffective in preventing the development of interstitial pneumonia in seronegative recipients who receive seropositive grafts. Furthermore, it is likely to be ineffective in CMV-seronegative recipients who receive CMV-seropositive blood products; however, the latter statement is not fully substantiated and a number of studies are in progress to delineate that point. It is likely that immunoglobulin is ineffective in CMV-seropositive recipients who receive either $\mathrm{CMV}$-seropositive or CMV-seronegative blood products.

We conducted our own trial with IVIG in which 119 patients were enrolled (7). Slightly more than half of the patients were CMV seronegative. The median observation time was 29 months. All the patients received $\mathrm{CMV}$-negative blood products and were given $500 \mathrm{mg} / \mathrm{kg}$ of IVIG every 2 weeks for eight administrations. There were no $\mathrm{CMV}$ infections and there was no CMV pneumonia in the first group of 57 patients, who were CMV seronegative and had received a graft from a CMVseronegative donor. In the next group of CMVseronegative recipients, who were given a graft from a CMV-seropositive donor, 3 of 12 patients developed viremia or viruria but none of them developed pneumonia. The low incidence of CMV interstitial pneumonia in this group indicated that IVIG might be effective in the CMV-seronegative patient even when receiving a seropositive donor graft. Unfortunately, IVIG was largely ineffective in seropositive patients. Of the 50 seropositive recipients, $33(66 \%)$ developed a positive CMV culture and 8 developed CMV pneumonia, which was fatal in 6 cases. When all groups are combined the overall effect of IVIG appears impressive: a $13 \%$ incidence of CMV infection and a $7 \%$ incidence of CMV pneumonia, suggesting that IVIG was able to reduce the incidence of CMV pneumonia, perhaps even in CMV-seropositive patients.

We tried to obtain more definitive information about the role of IVIG in seropositive recipients. 
We were unwilling to do a randomized, placebocontrolled study since we had ethical problems with not treating all our patients with IVIG. We speculated that if we were to give IVIG to seropositive patients in higher doses and more frequently, we might prevent not only CMV excretion (such as viremia or viruria) but also the development of CMV infection. For these reasons we limited our study to CMV-seropositive recipients and stratified them between two IVIG schedules, giving either $500 \mathrm{mg} / \mathrm{kg}$ every 2 weeks for 4 months or $500 \mathrm{mg} / \mathrm{kg}$ every week for 4 months. If patients became excretors of the virus, they received a dose of $500 \mathrm{mg} / \mathrm{kg}$ daily for 10 days. There were 24 patients in the standard group and 22 in the high-dose group. We observed no difference in the number of patients who became excretors- 15 in the standard-dose group and 17 in the high-dose group. There was no difference in the number of patients that developed systemic disease or in the number of fatalities observed. Thus, a higher dose of antibody does not appear to be more beneficial than a lower dose in preventing CMV disease. New strategies need to be developed to overcome the severe problem presented by the CMV-seropositive patient.

The treatment of established CMV pneumonia is not very effective. We have learned that ARA-A, acyclovir, and TFT are not effective in the therapy of this disease, although acyclovir might be effective as a preventive agent. IVIG alone is as effective as 9(1,3-dihydroxy-2-proxymethyl) guanine (DHPG) alone in treating established CMV pneumonia, but surprisingly, the combination of IVIG and DHPG is quite effective, and in a number of studies it has reduced the mortality rate from 85 to $50-60 \%(8,9)$.

Hemorrhagic gastroenteritis is the other major viral complication in the early postengraftment phase (10). The mortality is relatively high (29\%) even in the absence of acute GVHD. A number of viruses (such as rotavirus) are associated with hemorrhagic gastroenteritis. but recently CMV is becoming prevalent. About half of the cases of hemorrhagic gastroenteritis in our own patient population are now due to $\mathrm{CMV}$; the remainder are split between rotavirus and adenovirus. Effective measures to prevent this syndrome might include the following: strict isolation. effective prevention of GVHD (since GVHD might well set the stage for hemorrhagic gastroenteritis), and possibly therapy with orally administered immune globulin. There might be a rationale for such a therapy if we consider that the gastrointestinal tract is severely injured after transplantation. The preparative therapy as well as GVHD have caused severe mucosal injury. The Peyer's patches and other gutassociated lymphoid tissues have been destroyed and must be rebuilt. In the lamina propria IgA- and IgM-producing plasma cells have disappeared. Most importantly the degradation of IgG when administered orally is much impaired in such a devastated gut. It has been shown that $\mathrm{IgG}$ antibody survives passage through the gastrointestinal tract and emerges as an intact molecule. This led us to consider administering IVIG orally to patients at risk for hemorrhagic gastroenteritis.

We conducted a pilot trial in which 30 patients were given IVIG orally at a dose of $50 \mathrm{mg} / \mathrm{kg}$ for 4 weeks. Prior to administration of IVIG we could not demonstrate any IgG in the stool. After oral administration of IVIG, 20 of 30 patients had considerable amounts of IgG, up to $400 \mathrm{mg} / \mathrm{dl}$, in the stool. Eighteen patients had greater than $20 \mathrm{mg} / \mathrm{dl}$. Only 1 of the 30 patients developed a positive adenovirus culture and 12 patients remained afebrile (11). We have just completed a double-blind, randomized study comparing orally administered IVIG to placebo. Hopefully, the analysis of the study will show that the concept of oral administration of immune globulin has some merit.

The immunobiology of phase III, the late postengraftment phase, is characterized by a continuous impairment of the $T$ helper-cell function, a continuous defect in humoral immunity, and, in particular, the development of chronic GVHD. A number of infections are seen in this posttransplant phase. especially sinopulmonary infections with encapsulated organisms such as Streptococcus pneumoniae. Initially we thought that the hyposplenism associated with chronic GVHD was responsible for these infections (12). However, we considered that there might also be an association with an IgGsubclass deficiency and we conducted a small study. IgG subclasses were determined in 28 patients. One group of 14 patients showed a marked IgG2-subclass deficiency with a very high infection rate. Twelve of these 14 patients developed infections with gram-positive organisms. Another group of 14 patients with no IgG2-subclass deficiency had a very low infection rate, with only 2 of 14 being infected. Studying these patients further we could show that the patients continued to show subclass deficiency even after they resolved the infections. However, it is not only $\operatorname{IgG} 2$ but also IgG4 subclass which was markedly reduced (13). To prevent these 
late infections one could maintain coverage against gram-positive organisms beyond day 100 and should maintain gram-positive coverage for an even longer period in patients who demonstrate chronic GVHD. Most importantly, one should maintain IVIG supplementation beyond day 120 for the first year. In our transplant center we continue IVIG supplementation as long as the subclass deficiency persists, not unlike a patient with an inborn IgGsubclass deficiency syndrome. Utilizing this strategy one can virtually eliminate late gram-positive infections.

Since there are a number of sequential events in the immunobiology after transplantation which correlate with infectious patterns, it should be possible to develop rational strategies to counteract the various immune defects. The prophylactic and therapeutic use of IVIG might have a central role because it is likely to affect all phases of bone marrow transplantation. We know that immune globulins have a beneficial effect in both the prevention and the treatment of viral interstitial pneumonia. It is likely that immune globulin will also have a beneficial effect in the prevention of viral enteritis and in counteracting late subclass deficiency. There may also be some modulating influence on the entire immunobiology of bone marrow transplantation.

\section{REFERENCES}

1. Tutschka PJ, Copelan EA, Klein J: Bone marrow transplantation for leukemia following a new busulfan and cyclophosphamide regimen. Blood 70(5):1382-1388, 1987

2. Storb R, Prentice RL, Buckner CD, et al.: Graft versus host disease and survival in patients with aplastic anemia treated by marrow grafts from HLA-identical siblings: Beneficial effect of a protective environment. N Engl J Med 308:302307,1983

3. Munsler AM: Infections in burns. In Clinical Use of Intravenous Immunoglobulins. A Morell, UE Nydegger (eds). London, Academic Press, 1986. pp 339-344

4. Pollack M: Antibody therapy in gram-negative bacterial disease. In Clinical Use of Intravenous Immunoglobulins, A Morell, UE Nydegger (eds). London, Academic Press. 1986. pp 317-325

5. Pollard M. Chang CF. Srivastava KK: The role of microflora in development of graft versus host disease. Transplant Proc 8:533-536, 1976

6. Rossie KM, Sheridan JF. Barthold SW. Tutschka PJ: Graftvs-host disease and sialodacryoadenitis viral infection in bone marrow transplanted rats. Transplantation 45:10121016, 1988

7. Kapoor N, Copelan EA, Tutschka PJ: Cytomegalovirus (CMV) infection in bone marrow transplant recipients: Use of intravenous gammaglobulin as prophylactic and therapeutic agents. Transplant Proc XXI:3095-3096, 1989

8. Bratanow NC, Ash RC, Turner PA, et al.: Successful treatment of serious cytomegalovirus disease with $9(1,3-$ dihydroxy-2-propoxymethyl) guanine in bone marrow transplant (BMT) patients. Blood 68(5) Suppl 1:280a, 1986

9. Emanuel D, Peppard J, Chehimi J, Hammerling U, O'Reilly R: The diagnostic, prophylactic and therapeutic uses of monoclonal antibodies to human cytomegalovirus. Transplant Proc XIX(6) Suppl 7:132-137, 1987

10. Yolken RH. Bishop CA, Townsend TR, et al.: Infectious gastroenteritis in bone marrow transplant recipients. $N$ Engl J Med 306(17): 1009-1012, 1982

11. Copelan EA, Bechtel T, Featheringham NC, Grose MM, Sedmak DD, Kapoor N, Tutschka PJ: Oral administration of IgG in marrow transplant recipients. Drug Intell Clin Pharm 22:912. 1988

12. Al-Eid MD, Tutschka PJ, Wagner HN Ir, Santos GW, Tsan MF: Functional asplenia in patients with chronic graftversus-host disease: Concise communication. J Nucl Med 24:1123-1126, 1983

13. Sheridan JF, Tutschka PJ, Sedmak D. Copelan EA: IgG subclass deficiency and pneumococcal pneumonia following allogeneic marrow transplantation. Blood April(1)75/7:9093,1990

\section{DISCUSSION}

Dr. Gulati: What has been your experience with autologous transplants? Do you need to do all this with patients who are undergoing autologous transplants?

Dr. Tutschka: I do not think so. The incidence of interstitial pneumonia as well as hemorrhagic gastroenteritis is much reduced in autologous transplants-perhaps a quarter of that seen in allogeneic transplants. Furthermore, the immune system recovers much more readily after autologous bone marrow transplantation. On the other hand, autologous transplantation patients might provide an excellent target population in which to study more nonspecific effects, such as the reduction of infections during aplasia, because we do not have the compounding variables of GVHD, immune modulation phases, and so on. We are currently not using IVIG to the same extent in autologous transplants as we do in allogeneic transplants but $I$ think it is a good study population. 